HU-SEFT-1995-14

\title{
The $W_{R}$ production in $e \gamma$ collisions
}

\author{
K. Huitu \\ Research Institute for High Energy Physics, \\ University of Helsinki \\ J. Maalampi \\ Department of Theoretical Physics, University of Helsinki \\ M. Raidal| \\ Department of Theoretical Physics, University of Helsinki
}

February 8, 2020

\begin{abstract}
We consider the production of a single right-handed gauge boson $W_{R}$ in the high-energy $e^{-} \gamma$ collisions with polarized beams. If the associated neutrino is light, the reaction will give the best discovery reach for $W_{R}$ in the Next Linear Collider.
\end{abstract}

\footnotetext{
${ }^{1}$ On leave of absence from KBFI, Academy of Estonia; Address after October 1, 1995: Department of Theoretical Physics, University of Valencia, Spain
} 
The left-right symmetric model (LRM) [1] is an extension of the Standard Model (SM), in which the gauge interactions of left-handed and right-handed fundamental fermions are treated on equal basis. There could be reflections of this extended structure in low-energy phenomena due to possible mixings of the known particles with the new particles predicted by the model, such as deviations from the $\mathrm{V}$-A form of weak interactions, but nothing of that kind has turned up in experiments so far. The LRM is based on the gauge symmetry $S U(2)_{L} \times S U(2)_{R} \times U(1)_{B-L}$, and there are hence two new weak bosons, $W_{R}$ and $Z_{R}$, in addition to the ones known in the SM. The left-right symmetry, not present in the low energy world, is broken by a $\mathrm{SU}(2)_{R}$ triplet Higgs field $\Delta=\left(\Delta^{++}, \Delta^{+}, \Delta^{0}\right)$. The only new fermions the model predicts are the right-handed neutrinos.

The energy scale $v_{R}=\left\langle\Delta^{0}\right\rangle$ of the breaking of the LRM symmetry to the SM symmetry, which also sets, up to coupling constants, the mass scale of the new weak bosons and right-handed neutrinos, is not given by the theory itself. Its value can only be deduced from experimental data. There exist several indirect bounds from processes where some of the new particles appear as virtual states, as well as direct discovery limits. According to these results it is not excluded that the left-right symmetry manifests itself already at the scale of $O(1) \mathrm{TeV}$. Hence, the search for direct evidences of the leftright symmetry is a relevant issue to be considered in connection with the Next Linear Collider (NLC).

In this note we shall investigate one particular reaction possible in NLC, namely the production of the right-handed weak boson $W_{2}$ (we denote this particle $W_{2}$ instead of $W_{R}$ as there may be a slight mixing between $W_{R}$ and $W_{L}$ ) and a neutrino in the electron-photon collision (see also discussion in [2])): 


$$
e^{-}+\gamma \rightarrow W_{2}+N
$$

Here $N$ can be either a heavy right-handed neutrino $\nu_{R}$ or, in the case of a large neutrino mixing, an ordinary light neutrino $\nu_{L}$. So far, the $e^{-} \gamma$ collisions have been studied using the photon spectrum of classical Bremsstrahlung. In the linear collider it will be possible to obtain high luminosity photon beams by backscattering intensive laser pulses off the electron beam [3] without considerable losses in the beam energy and with very high polarizability and monochromaticity [4].

If the neutrino is light, the reaction (11) would be the energetically most favourable place to produce $W_{2}$. It has clean signatures; if neutrino does not decay in the detector, the signal will be two energetic jets and missing energy, or a single lepton and missing energy. Using polarized beams will significantly reduce backgrounds. The reaction would be useful in yielding valuable information on both the gauge boson and neutrino masses.

Let us first discuss the existing bounds on the mass $M_{W_{2}}$ of the new charged boson. In the Tevatron one has made a direct search of $W_{2}$ in the channel $p p \rightarrow W_{2} \rightarrow e N$. The bound they give is $M_{W_{2}} \gtrsim 652 \mathrm{GeV}$ [5]. The result is based on several assumptions on the LRM: the quark- $W_{2}$ coupling has the SM strength, the CKM matrices for the left-handed quarks and the right-handed quarks are similar, the right-handed neutrino does not decay in the detector but appears as missing $E_{T}$. If one relaxes the first two assumptions, the mass bound will be weakened considerably, as was pointed out in [6]. The third assumption is also crucial; if the right-handed neutrino is heavy, with a mass of say $100 \mathrm{GeV}$ or more, it will decay in the detector into charged particles (one possible channel could be $\nu_{R} \rightarrow l+q \overline{q^{\prime}}$ ) with no missing energy. For this case the Tevatron search would be ineffective.

If the right-handed neutrino is heavier than $W_{2}$, the reaction considered in 
the Tevatron search is forbidden altogether if there is no substancial mixing between left- and right-handed neutrinos. In this case $W_{2}$ could decay only to non-leptonic states. Obviously, in the Tevatron hadronic signals are much more difficult to identify than leptonic ones, and therefore the mass limit obtained from hadronic decay channels is not particularly restrictive.

There are not very definite mass limits for the right-handed Majorana neutrinos. At low energies they are assumed to be almost sterile; they do couple to the ordinary $W$ and $Z$ bosons due to their mixing with the lefthanded neutrinos [7]. This mixing is, however, quite small, of the order of $m_{l, q} / m_{R}$, where $m_{l, q}$ is a charged lepton or quark mass and $m_{R}$ is the mass scale of the breaking of the left-right symmetry. It is also possible that the ordinary weak bosons contain a small fraction of $W_{R}$ and $Z_{R}$ states, which would allow the right-handed neutrinos to couple to them, but also such mixings are known to be small [8]. Hence the laboratory mass limits for the right-handed neutrinos are not that stringent.

Cosmological constraint on the mass of the right-handed neutrinos derives from the condition that the energy density associated with them should not exceed the estimated total energy density of the Universe. It was shown in [9] that if the mixing between the left-handed and right-handed sectors is on the level of 0.1 , the mass of the right-handed neutrino should exceed $30 \mathrm{GeV}$.

The conclusion from above is that there is still some uncertainty in the mass bounds on the $W_{2}$ and the right-handed neutrinos. It was argued in [6], that the lower limit for $M_{W_{2}}$ could be as low as $300 \mathrm{GeV}$.

We wish to emphasize the usefulness of the reaction (11) in studying the production and the mass relation of $W_{2}$ and the right-handed neutrinos $\nu_{R}$ (the right-handed electron neutrino if neutrinos of different families do not mix). This reaction has advantages compared with the corresponding processes in hadron colliders, such as Tevatron and LHC. At NLC, good heavy 
flavour tagging would make the detection of $W_{2}$ and $\nu_{R}$ efficient. Also, here the $W_{2}$ production is insensitive to the unknown CKM matrix of the righthanded quarks. In addition, the possibility of having highly polarized electron and photon beams provides new probes for testing various properties of the new particles [10].

There are two Feynman diagrams contributing at the tree level to reaction (四) (see Fig. 1). The helicity amplitudes of the reaction for general V,A -interactions are given in ref. [11]. In determining the cross sections from the helicity amplitudes we have assumed $100 \%$ polarized electron and photon beams. This is, of course, an approximation, since in practice the polarizations will never be ideal and one has to employ a density matrix giving the polarization parameters of the beams.

The mass dependence of the total cross section of the process $e_{R}^{-} \gamma \rightarrow$ $W_{2}^{-} N$ can be seen in Fig. 2, where we plot the cross section as a function of $W_{2}^{-}$mass for the center of mass energy $\sqrt{s_{e \gamma}}=1.5 \mathrm{TeV}$, expected to be possible to achieve in the final stage of NLC, assuming the left- (Fig. 2 (I)) and right-handedly (Fig. 2 (II)) polarized photon beams. The curves denoted by $a$ and $b$ correspond to the neutrino masses $M_{N}=300 \mathrm{GeV}$ and $M_{N}=600$ $\mathrm{GeV}$, respectively. The cross sections are found to be reasonably large for almost the entire kinematically allowed mass region, decreasing faster with $M_{W_{2}}$ for the $\tau_{1}=1$ photons. At low $W_{2}$ masses the difference between $a$ and $b$ curve is small but for heavy $W_{2}$ masses the cross section depends strongly on the neutrino mass. If $M_{N} \leq M_{W_{2}}$, reaction (1) enables us to study heavier vector bosons than what is possible in the $W_{2}^{-}$pair production in $e^{-} e^{+}$or $e^{-} e^{-}$collisions.

The reaction would be even more useful in this respect if the mixing between the heavy and the light neutrino is large enough to give observable effects. In Fig. 3 we plot the cross section of the reaction $e_{R}^{-} \gamma \rightarrow W_{2}^{-} \nu$ 
for different photon polarizations assuming a vanishing mass of $\nu$ and the neutrino mixing angle of $\sin \theta_{N}=0.05$. For this set of parameters the process should be observable up to $W$-boson mass $M_{W}=1.2 \mathrm{TeV}$.

In order to see the relative importance of different polarization states we present in Fig. 4 the angular distributions of the differential cross sections for all combinations of polarizations. In Fig. 4 (I) we plot the differential cross sections for the left-handedly polarized photon beam assuming the collision energy $\sqrt{s_{e \gamma}}=1.5 \mathrm{TeV}$, the gauge boson mass $M_{W_{2}}=700 \mathrm{GeV}$ and the neutrino mass $M_{N}=300 \mathrm{GeV}$. In Fig. 4 (II) we plot the same for the righthandedly polarized photon beam. As the figures show, the final states with a left-handed neutrino are clearly suppressed. The main part of the cross section is again coming from the case where the photon and $W^{-}$are polarized in the same way. The other polarization combinations are somewhat suppressed for the right-handedly polarized photon beam. While in the SM all differential cross sections are peaked in the backward direction, in this case the distributions are more flat for many polarization states providing better possibilities for detecting the anomalous photon coupling.

In conclusion, we have pointed out the usefulness of the electron photon collisions in testing the left-right symmetry of electroweak interactions at high energies through the reaction $e^{-} \gamma \rightarrow W_{2} N$. If the right-handed neutrino is light, this reaction offers a much better discovery reach for $W_{2}$ than the pair production in $e^{+} e^{-}$or $e^{-} e^{-}$collisions.

Acknowledgements. We are indebted to Aarre Pietil, Turku, for useful discussions. One of us (M.R.) expresses his gratitude to Emil Aaltosen Säätiö, Wihurin Rahasto and Visln Rahasto for grants. This work has been supported by the Academy of Finland. 


\section{References}

[1] J.C. Pati and A. Salam, Phys. Rev. D 10 (1974) 275;

R.N. Mohapatra and J.C. Pati, Phys. Rev D 11 (1975) 566, 2558;

G. Senjanovic and R.N. Mohapatra, Phys. Rev. D 12 (1975) 1502.

[2] A. Pietil, talk given in First Arctic Workshop on Future Physics and Accelerators (Saariselkä, Finland, August 21-26, 1994), to appear in proceedings.

[3] I. Ginzburg, G. Kotkin, V. Serbo and V. Telnov, Pizma ZhEFT 34 (1981) 514 and Nucl. Instrum. Methods 205 (1983) 47.

[4] V. Telnov, talks given in Gamma-Gamma Collider Workshop (Berkeley, USA, March 28-31, 1994) and First Arctic Workshop on Future Physics and Accelerators (Saariselkä, Finland, August 21-26, 1994), to appear in proceedings.

[5] F. Abe et al., Phys. Rev. Lett. 74 (1995) 2900.

[6] T. Rizzo, Phys. ReV. D 50 (1994) 325, and ibid. 5602.

[7] M. Gronau, Phys. Rev. D 29 (1984) 2539.

[8] J.F. Gunion, J. Grifols, A. Mendes, B. Kayser, and F. Olness, Phys. Rev. D40 (1989) 1546; N.G. Deshpande, J.F. Gunion, B. Kayser, and F. Olness, Phys. Rev. D44 (1991) 837.

[9] K. Enqvist, K. Kainulainen and J. Maalampi, Nucl. Phys. B316 (1989) 456.

[10] D. Choudhury and F. Cuypers, Phys. Lett. B 325 (1994) 500 and Nucl. Phys. B 429 (1994) 33; P. Helde, K. Huitu, J. Maalampi and M. Raidal, 
Nucl. Phys. B 437 (1995) 305; F. Cuypers, K. Kolodziej and R. Rückl, preprint MPI-PhT/94-33.

[11] M. Raidal, Nucl. Phys. B 441 (1995) 49. 


\section{Figure captions}

Fig. 1. Feynman diagrams for the process $e^{-} \gamma \rightarrow W^{-} N$.

Fig. 2. The total cross section of the process $e_{R}^{-} \gamma \rightarrow W_{2}^{-} N$ as a function of heavy gauge boson mass for the left- (figure (I)) and right-handedly (figure (II)) polarized photon beams. The collision energy is taken to be $\sqrt{s_{e \gamma}}=1.5 \mathrm{TeV}$, and the mass of heavy neutrino $M_{N}=300 \mathrm{GeV}$ and $M_{N}=600 \mathrm{GeV}$ for curves $a$ and $b$, respectively.

Fig. 3. The total cross section of the process $e_{R}^{-} \gamma \rightarrow W_{2}^{-} \nu$ as a function of heavy gauge boson mass for the left- and right-handedly polarized photon beams. The collision energy is taken to be $\sqrt{s_{e \gamma}}=1.5 \mathrm{TeV}$ and the neutrino mixing angle $\sin \theta=0.05$.

Fig. 4. The angular distributions of differential cross sections of various $N W_{2}^{-}$polarization states for the left- (figure (I)) and right-handedly (figure (II)) polarized photon beams in the case of left-right model. The collision energy is taken to be $\sqrt{s_{e \gamma}}=1.5 \mathrm{TeV}$, the mass of right handed vector boson $M_{W}=700 \mathrm{GeV}$ and the mass of heavy neutrino $M_{N}=300 \mathrm{GeV}$. The number pairs in figure denote the helicity states of neutrino and gauge boson, respectively. 

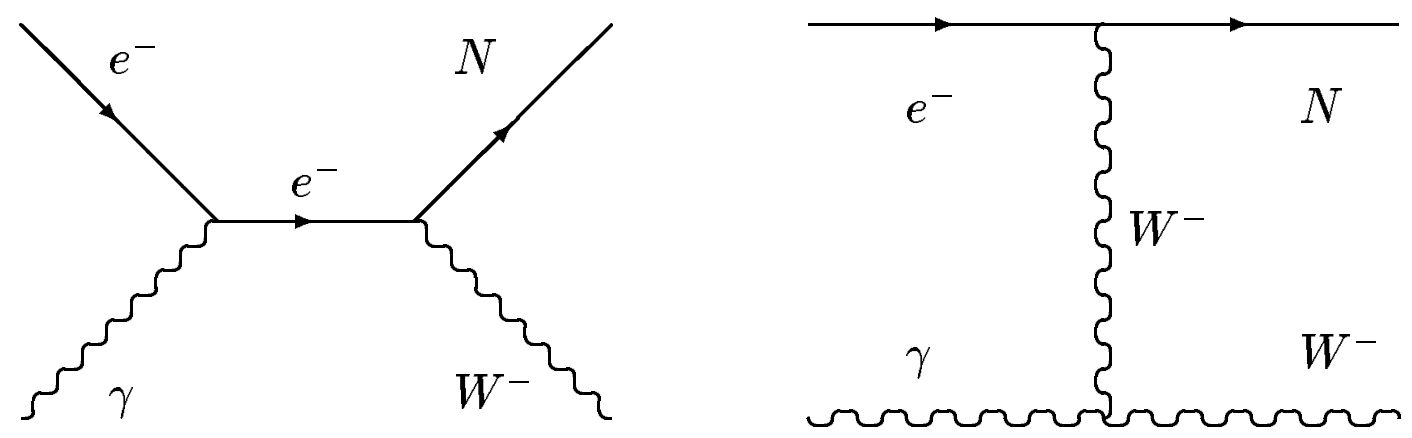

Figure 1: Feynman diagrams for the process $e^{-} \gamma \rightarrow W^{-} N$. 


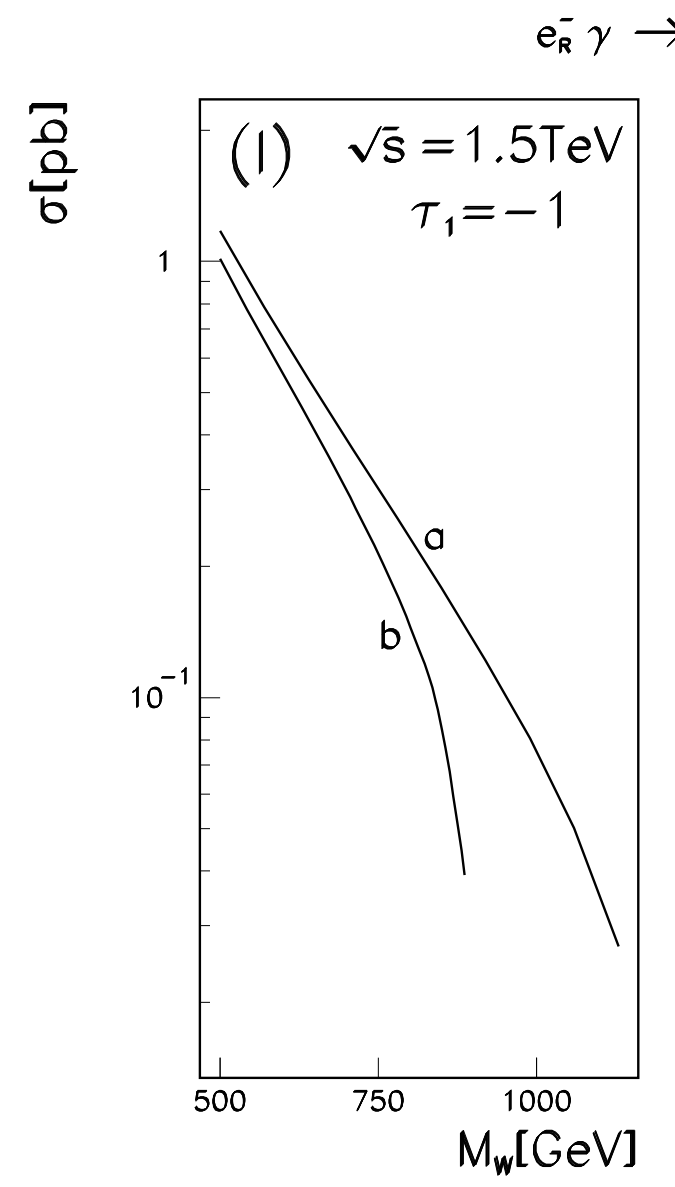

$\mathrm{W}_{2}^{-} \mathrm{N}$

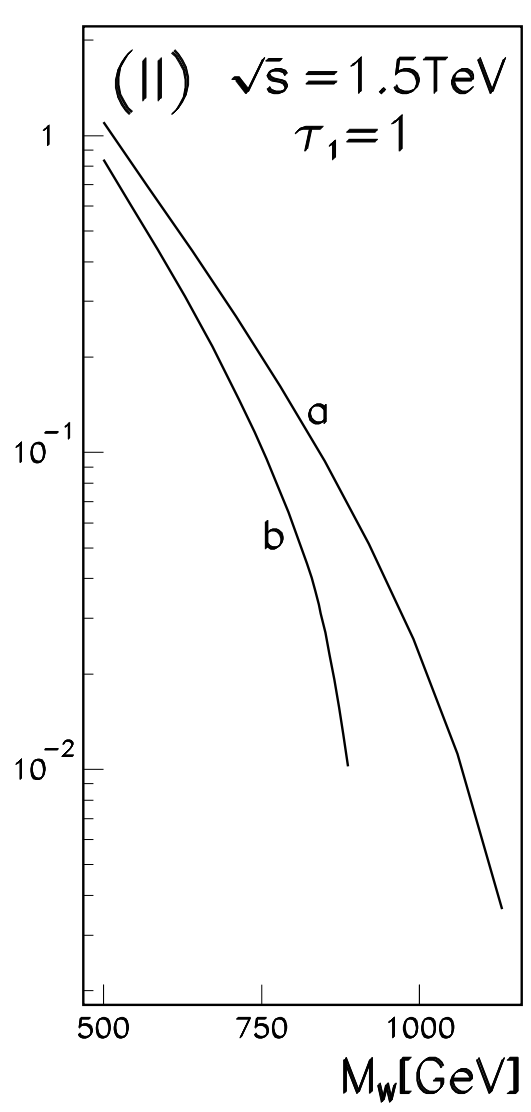

Figure 2: The total cross section of the process $e_{R}^{-} \gamma \rightarrow W_{2}^{-} N$ as a function of heavy gauge boson mass for the left- (figure (I)) and right-handedly (figure (II)) polarized photon beams. The collision energy is taken to be $\sqrt{s_{e \gamma}}=1.5$ $\mathrm{TeV}$, the mass of heavy neutrino $M_{N}=300 \mathrm{GeV}$ and $M_{N}=600 \mathrm{GeV}$ for curves $a$ and $b$, respectively. 


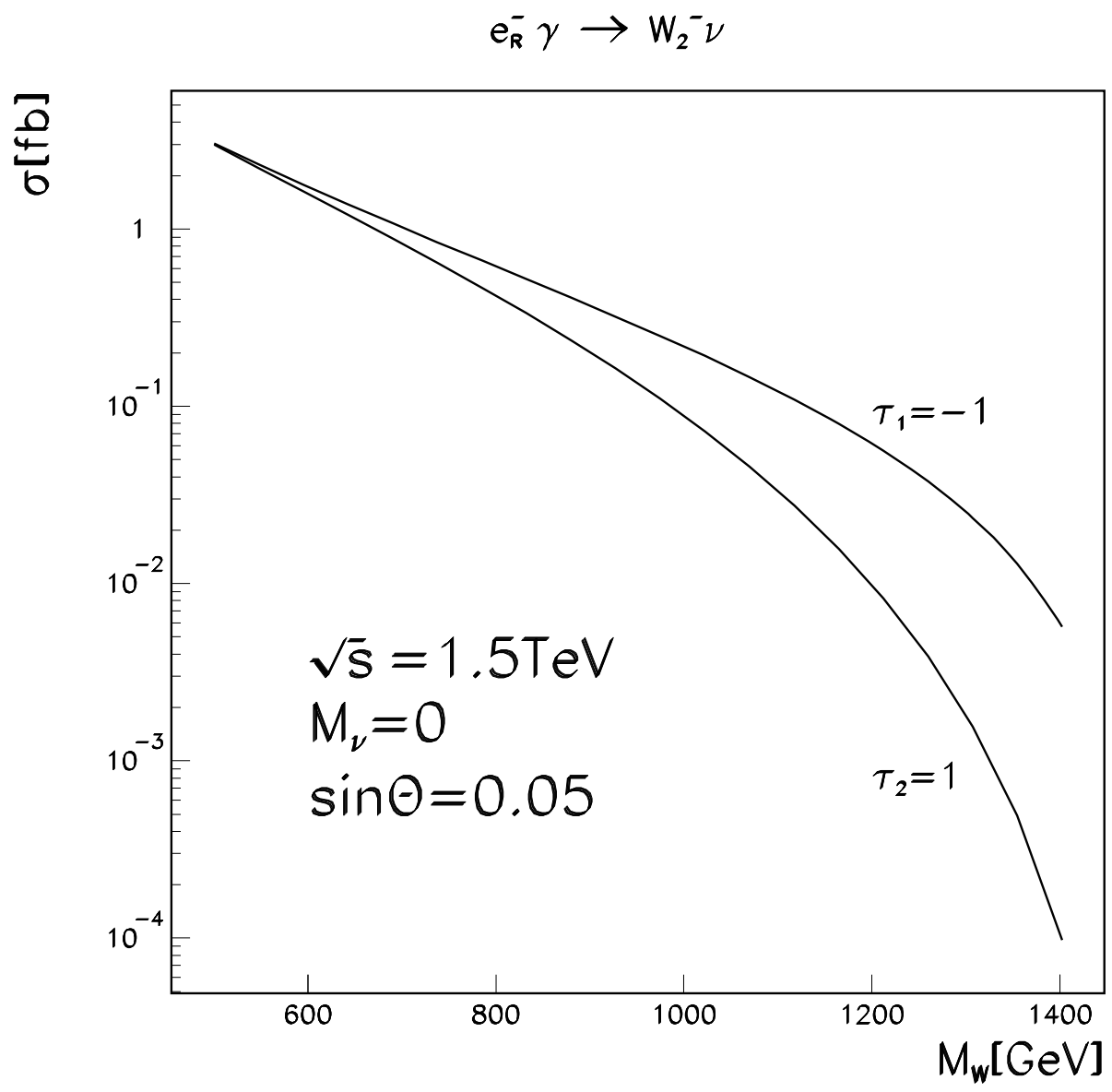

Figure 3: The total cross section of the process $e_{R}^{-} \gamma \rightarrow W_{2}^{-} \nu$ as a function of heavy gauge boson mass for the left- and right-handedly polarized photon beams. The collision energy is taken to be $\sqrt{s_{e \gamma}}=1.5 \mathrm{TeV}$ and the neutrino mixing angle $\sin \theta=0.05$. 


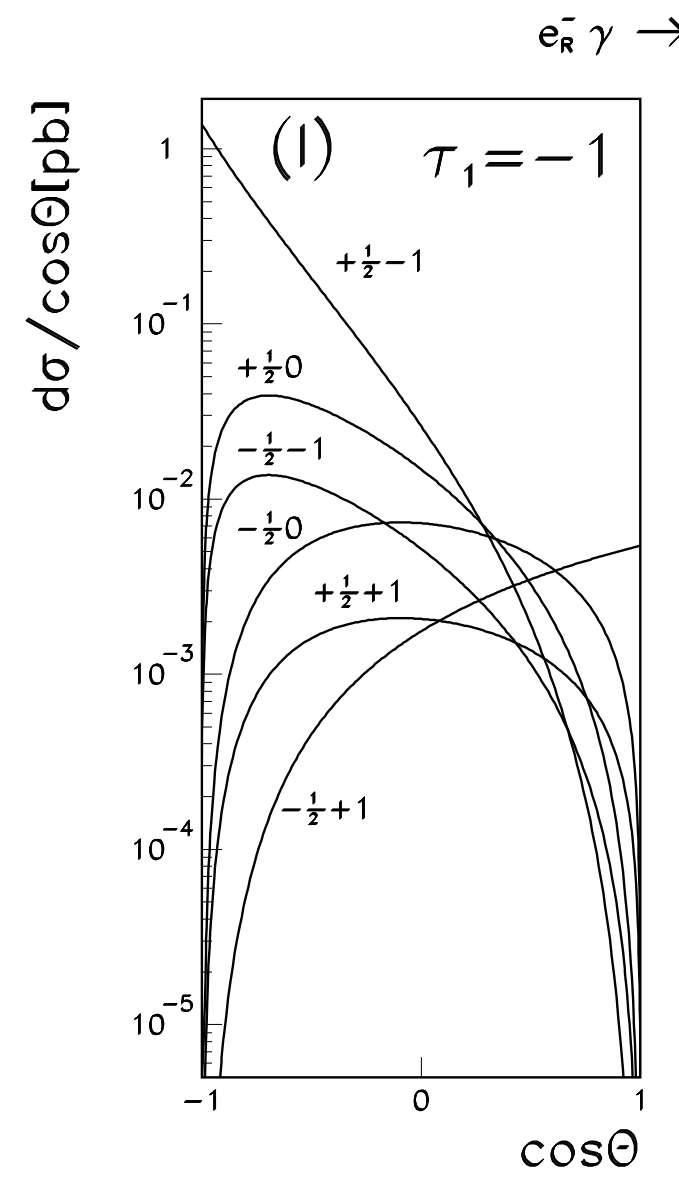

$\mathrm{W}_{2}^{-} \mathrm{N}$

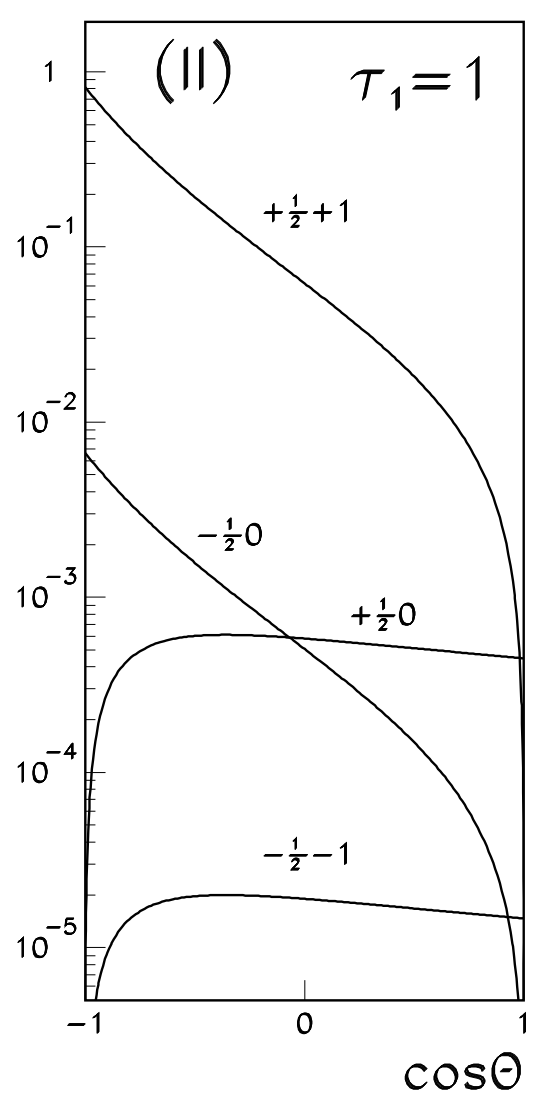

Figure 4: The angular distributions of differential cross sections of various $\mathrm{N}_{2}^{-}$polarization states for the left- (figure (I)) and right-handedly (figure (II) ) polarized photon beams in the case of left-right model. The collision energy is taken to be $\sqrt{s_{e \gamma}}=1.5 \mathrm{TeV}$, the mass of right handed vector boson $M_{W}=700 \mathrm{GeV}$ and the mass of heavy neutrino $M_{N}=300 \mathrm{GeV}$. The number pairs in figure denote the helicity states of neutrino and gauge boson, respectively. 